DE

M E D I C I N A

T R O P I C A L

$\mathrm{DE}$

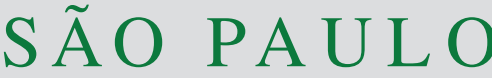

JOURNAL OF THE SÃO PAULO INSTITUTE OF TROPICAL MEDICINE

(1)Universidad de Valencia, Facultad de Farmacia, Departamento de Parasitología, Valencia, España

(2)Universidade Federal do Paraná, Setor de Ciências Biológicas, Departamento de Patologia Básica, Curitiba, Brazil

Correspondence to: Carla Muñoz-Antoli Universidad de Valencia, Facultat de Farmacia, Departamento de Parasitología, Avda. Vicent Andrés Estellés, s/n, 46100, Burjassot, Valencia, España

E-mail: carla.munoz@uv.es

Received: 6 June 2017

Accepted: 28 August 2017

\section{Intestinal symptoms and Blastocystis load in schoolchildren of Paranaguá Bay, Paraná, Brazil}

\author{
Raimundo Seguí ${ }^{1}$, Debora Klisiowicz², Camila Yumi Oishi ${ }^{2}$, Rafael Toledo ${ }^{1}$, \\ José Guillermo Esteban ${ }^{1}$, Carla Muñoz-Antoli' ${ }^{1}$
}

\section{ABSTRACT}

The symptomatology of Blastocystis cannot be attributed to any particular subtype, although can be related to a high Blastocystis infection load. One stool sample from each of 217 schoolchildren of Vale de Sol Paranaguá Bay (Paraná, Brazil) was collected. Three milliliters of each fixed stool sample were processed applying the formalin-ether concentration technique. After obtaining the overall prevalence of intestinal parasites, quantification was carried out in Blastocystis positive samples. A total of 75/217 (34.6\%) children suffered from intestinal symptoms (abdominal pain and/or persistent diarrhea), of whom $41.3 \%$ (31/75) presented moderate/heavy Blastocystis load with a statistically significant risk to present intestinal symptoms (OR 0.039 [0.006-0.15]; $\mathrm{p}<0.001$ ) Moreover, those symptomatic schoolchildren monoparasitized only by Blastocystis $(10 / 75,13.3 \%)$, and those polyparasitized by Blastocystis with other non-pathogenic species $(15 / 75,20 \%)$ with moderate/heavy loads, also entail a statistically significant risk of intestinal symptomatology, both in monoparasitism (12\%, OR 0.10 [0.004-0.63]; $\mathrm{p}=0.021)$ and in polyparasitism with a non-pathogenic parasite $(18.6 \%$, OR 0.059 [0.002-0.35]; p=0.001). For the first time in Brazil, using data from schoolchildren of Paranaguá Bay, we demonstrated that moderate/ heavy loads of Blastocystis could be related to intestinal symptoms.

KEYWORDS: Blastocystis spp. Intestinal symptoms. Load. Schoolchildren intestinal parasitism. Parasite load. Parasite burden.

Intestinal parasite infection decreases quality of life and increases susceptibility to other infections ${ }^{1}$. Blastocystis infects between 1-2 billion people on a global scale $^{2}$. Blastocystis prevalence in humans varies from $30-76 \%$ in developing countries $^{3}$. The role of Blastocystis in human health and disease remains unclear. Although commonly represented by asymptomatic infections, it does not mean that Blastocystis is not pathogenic, which is a similar situation found in Giardia intestinalis infections ${ }^{4}$.

In patients with negative results for other potentially pathogenic microorganisms, the symptomatology of Blastocystis could not be attributed to any particular subtype ${ }^{5}$. However, it can be related to hosts' related factors such as host genetics, immune status, host response/stress, concomitant infections, dysbiosis of gut microbiota, etc. A high Blastocystis load could also be considered the cause of the patient's intestinal symptomatology $y^{2,6}$. Herein, for the first time in Brazil, the relationship between intestinal symptoms and the Blastocystis load in schoolchildren of Paranaguá Bay (Paraná, Brazil) is examined.

Each of the 217 recruited schoolchildren, aged 1-15 years, from Vale de Sol, a coastal city of Paranaguá (Brazil), provided a stool sample, which was collected 
in a plastic container. Stool samples were fixed in $10 \%$ formalin using a $1 / 3$ proportion. Informed consent was obtained from each participant's legal guardian before the recruitment following the instructions of Comité Ético de la Universitat València (H1431958278890).

Three milliliters of each fixed stool sample were processed using the formalin-ether concentration technique ${ }^{7}$. The entire sediment was examined to establish the intestinal parasite prevalence. Samples were considered negative when no parasite structures were observed in the entire sediment. In addition, in positive Blastocystis samples, quantification was carried out in the entire sediment based on the number of parasite structures observed at a magnification of 400x following Speich et al. ${ }^{8}$ with some modifications. Briefly, a total of five fields per slide were observed and an absolute number was assigned for categorizing Blastocystis loads as follows: a) one to four Blastocystis structures per slide were observed ( 5 fields); b) one parasite structure per field was observed; c) > one parasite structure per field was observed. The mean number after all observations was calculated, considering: a) low infection load; b) moderate infection load; and c) heavy infection load. Percentages of each infection load were calculated.

Statistical analysis was performed using the Open Source Epidemiologic Statistics for Public Health, version 3.03a. . The odds ratios (OR) and 95\% confidence interval of those having symptoms and being infected with low loads of Blastocystis were compared to the ones from patients with moderate and/or heavy loads, were calculated using a logistic regression. The level of statistical significance was $\mathrm{p}<0.05$.

In the entire study, an overall prevalence of intestinal infection of $43.8 \%(95 / 217)$ was found. No statistical differences were noticed regarding sex, but the group composed of 5-9 year old children was the most infected $\mathrm{p}<0.001$. A total of eight protozoan and three helminth species were detected (Table 1), with Blastocystis being the most prevalent $(31.8 \%)$. The low prevalence of soil transmitted helminth species was noteworthy.

From the total of 217 schoolchildren, 34.6\% (75/217) suffered from intestinal symptoms (abdominal pain and/or persistent diarrhea). Among them, 44\% (33/75) were parasitized by Blastocystis, and only $2.7 \%$ (2/75) presented a low parasite load (Table 2), which suggests that the parasitism caused by Blastocystys by means of a moderate to heavy load $(41.3 \%, 31 / 75)$ represent a statistically significant risk of the onset of symptoms (OR 0.039 [0.006-0.15]; $\mathrm{p}<0.001$ ).

Due to the detection of other pathogenic intestinal parasites (Table 1), in order to rule out their possible involvement with intestinal symptoms, symptomatic
Table 1 - Prevalence of intestinal parasite species in the 217 schoolchildren studied in Paranaguá Bay (Paraná, Brazil)

\begin{tabular}{lc}
\hline Parasites & $\begin{array}{c}\mathrm{N}=217 \\
(95 \% \mathrm{C} . \mathrm{I} .)^{*}\end{array}$ \\
\hline PROTOZOA & $43,3(36,8-50)$ \\
Blastocystis spp. & $31,8(25,9-38,2)$ \\
Endolimax nana & $13,4(9,3-18,4)$ \\
Giardia intestinalis & $12,9(8,9-17,9)$ \\
Entamoeba hartmanni & $5,1(2,7-8,6)$ \\
Entamoeba complex\# & $2,8(1,1-5,7)$ \\
Entamoeba coli & $2,3(0,8-5)$ \\
lodamoeba butschlii & $2,3(0,8-5)$ \\
Chilomastix mesnili & $0,9(0,2-3)$ \\
HELMINTHS & $7,8(4,8-12)$ \\
Ascaris lumbricoides & $6,5(3,7-10,3)$ \\
Trichuris trichiura & $0,9(0,2-3)$ \\
Ancylostomatidae & $0,5(0-2,3)$ \\
\hline TOTAL & $43,8(37,3-50,4)$ \\
\hline
\end{tabular}

* \%= percentage; $95 \%$ C.I.=95\% confidence interval. "Entamoeba complex= Entamoeba histolytica/E. dispar/ E. moshkovskii

Table 2 - Blastocystis loads in symptomatic schoolchildren studied in Paranaguá Bay (Paraná, Brazil)

\begin{tabular}{lcc}
\hline & \multicolumn{2}{c}{ Intestinal Symptoms N=75 } \\
\cline { 2 - 3 } & Low (\%) & Moderate-Heavy (\%) \\
\hline Blastocystis & $2(2.7)$ & $31(41.3)$ \\
Monoparasitism & $1(1.3)$ & $9(12)$ \\
Polyparasitism (non-pathogen) & $1(1.3)$ & $14(18.6)$ \\
\hline
\end{tabular}

schoolchildren monoparasitized only by Blastocystis $(13.3 \%, 10 / 75)$, and those polyparasitized by Blastocystis and other non-pathogenic species $(20 \%, 15 / 75)$ were selected.

A moderate to heavy Blastocystis load (Table 2) entails a statistically significant risk of intestinal symptomatology, both in monoparasitism (12\%, OR 0.10 [0.004-0.63]; $\mathrm{p}=0.021$ ), as well as in polyparasitism with a nonpathogenenic parasite (18.6\%, OR 0.059 [0.002-0.35]; $\mathrm{p}=0.001$ ).

Classifying an organism as pathogenic is not always straightforward ${ }^{2}$. The pathogenic potential of Blastocystis is associated with non-specific gastrointestinal symptoms, such as diarrhea, nausea, vomiting, abdominal pain and irritable bowel syndrome ${ }^{9,10}$, having direct and indirect effects on the human gut. The direct effects, triggered by the adherence to the gut epithelium and mediated by cystein proteases and secretion of the diarrheagenic toxins 
as virulence factors ${ }^{11}$, include apoptosis, degradation of tight-junction proteins, increased intestinal permeability, induction of pro-inflammatory cytokines and downregulation of iNOS. The indirect pathogenic effects are immunomodulatory properties that facilitate other enteric pathogens and produce alterations in the microbiota ${ }^{9}$.

Not all humans are susceptible toinfections caused by Blastocystis, and this parasite can be detected in healthy asymptomatic hosts. However, data from schoolchildren of Paranaguá Bay demonstrate that moderate/heavy Blastocystis loads could be related to intestinal symptoms. Blastocystis does not only colonize, but also multiply in the intestine. There seems to be a need for certain intestinal conditions for this replication to take place without braking and/or control. Thus, in a favorable environment, the Blastocystis load can increase and reach levels that may lead to harmful effects, with appearance of symptoms.

This study was affected by three limitations: first, multiple stool samples should be examined per child; second, stool samples were not tested for bacteria, viruses or fungus and, therefore, such etiologies cannot be ruled out; and third, more sensitive molecular approaches should be added to the research so as to improve diagnostic accuracy and allow determination of the subtype of Blastocystis involved. In this sense, this study represents the first step towards a more comprehensive study to clarify the clinical significance of Blastocystis in Paranaguá, Brazil.

\section{ACKNOWLEDGEMENTS}

Beca Erasmus Mobilitat Internacional de Doctorat 20142015; Secretaria de Educação e de Saúde de Paranaguá (Paraná, Brazil); Coproparasitological material was provided by DURVIZ S.L.; Project No. RD12/0018/0013, RICET, IV National I+D+I Program 2008-2011, ISCIIISubdirección General de Redes y Centros de Investigación Cooperativa and FEDER, Ministry of Health and Consumption, Madrid, Spain.

\section{AUTHORS' CONTRIBUTIONS}

CMA created and designed the study protocol; RS, DK and CYO carried out the sampling collection; RS and CMA carried out the data analysis and interpretation; CMA and RS drafted the manuscript; RT and JGE critically revised the manuscript. All authors read and approved the final manuscript.

\section{REFERENCES}

1. Maçin S, Kaya F, Çağdaş D, Hizarcioglu-Gulsen H, Saltik-Temizel IN, Tezcan İ, et al. Detection of parasites in children with chronic diarrhea. Pediatr Int. 2016;58:531-3.

2. Scanlan PD, Stensvold CR. Blastocystis: getting to grips with our guileful guest. Trends Parasitol. 2013;29:523-9.

3. Osman M, El Safadi D, Cian A, Benamrouz S, Nourrisson C, Poirier $\mathrm{P}$, et al. Prevalence and risk factors for intestinal protozoan infections with Cryptosporidium, Giardia, Blastocystis and Dientamoeba among schoolchildren in Tripoli, Lebanon. PLoS Negl Trop Dis. 2016;14;10:e0004496.

4. Stensvold CR, Clark CG. Current status of Blastocystis: a personal view. Parasitol Int. 2016;65:763-71.

5. Ramírez JD, Flórez C, Olivera M, Bernal MC, Giraldo JC. Blastocystis subtyping and its association with intestinal parasites in children from different geographical regions of Colombia. PLoS One. 2017;12:e172586.

6. Pavanelli MF, Kaneshima EN, Uda CF, Colli CM, FalavignaGuilherme AL, Gomes ML. Pathogenicity of Blastocystis sp. to the gastrointestinal tract of mice: relationship between inoculum size and period of infection. Rev Inst Med Trop Sao Paulo. 2015;57:467-72.

7. Knight WB, Hiatt RA, Cline BL, Ritchie LS. A modification of the formol-ether concentration technique for increased sensitivity in detecting Schistosoma mansoni eggs. Am J Trop Med Hyg. 1976;25:818-23.

8. Speich B, Marti H, Ame SM, Ali SM, Bogoch II, Utzinger J, et al. Prevalence of intestinal protozoa infection among school-aged children on Pemba Island, Tanzania, and effect of single-dose albendazole, nitazoxanide and albendazole-nitazoxanide. Parasit Vectors. 2013;6:3

9. Ajjampur SS, Tan KS. Pathogenic mechanisms in Blastocystis spp. - interpreting results from in vitro and in vivo studies. Parasitol Int. 2016;65:772-9.

10. Dogruman-Al F, Kustimur S, Yoshikawa H, Tuncer C, Simsek Z, Tanyuksel M, et al. Blastocystis subtypes in irritable bowel syndrome and inflammatory bowel disease in Ankara, Turkey. Mem Inst Oswaldo Cruz. 2009;104:724-7.

11. Grazyk TK, Shiff CK, Tamang L, Munsaka F, Beitin AM, Moss WJ. The association of Blastocystis hominis and Endolimax nana with diarrheal stools in Zambian school-age children. Parasitol Res. 2005;98:38-43. 